\title{
Sarcoma indiferenciado primario de corazón. A propósito de un caso
}

\author{
Juan Daniel Díaz-García', Fabián Armando Carrera-Patiño', Jorge Eduardo Aquino-Matus', Erika Karina Tenorio-Aguirre', \\ Haydee Viridiana Martínez-Hernández² \\ 'Servicio de Medicina Interna. Hospital General Dr. Manuel Gea González. Secretaría de Salud. Ciudad de México. México \\ ¿2Servicio de Anatomía Patológica. Hospital General Dr. Manuel Gea González. Secretaría de Salud. Ciudad de México. México
}

Recibido: 15/09/2018

Aceptado: 02/01/2019

En línea: 30/04/2019

Citar como: Díaz-García JD, Carrera-Patiño FA, Aquino-Matus JE, Tenorio-Aguirre EK, Martínez-Hernández HV. Sarcoma indiferenciado primario de corazón. A propósito de un caso. Rev Esp Casos Clin Med Intern (RECCMI). 2019 (Abr); 4(1): 6-9 doi: 10.32818/reccmi.a4n1a3.

Autor para correspondencia: Juan Daniel Díaz-García.judan_digar@hotmail.com

\section{Palabras clave \\ $\triangleright$ Neoplasia cardíaca \\ $\triangleright$ Sarcoma \\ $\triangleright$ Disnea \\ $\triangleright$ Hemoptisis \\ $\triangleright$ Síndrome constitucional}

\begin{abstract}
Resumen
Los tumores primarios de corazón son una entidad muy infrecuente, con una incidencia del $0.00-0.02 \%$ en series de autopsia. Aproximadamente un 15\% de estos tumores son malignos, siendo éstos extremadamente raros (menos del $0.1 \%$ ) y en su mayoría sarcomas. El diagnóstico se realiza habitualmente con técnicas de imagen como ecocardiograma, tomografía computarizada o resonancia magnética. El tratamiento de primera línea es la resección quirúrgica completa del sarcoma y el pronóstico es pobre en casi todos los casos. Se presenta el caso de un paciente masculino de 67 años de edad que comienza con cuadro de tos productiva, hemoptisis, disnea y síndrome constitucional. Con diagnóstico post mórtem de sarcoma indiferenciado primario de corazón.

Abstract
Primary heart tumors are a very infrequent entity, with an incidence of 0.0017 to $0.02 \%$ in autopsy series. Ap-
proximately $15 \%$ of these tumors are malignant, being these extremely rare (less than 0.1\%) and mostly sar-
comas. The diagnosis is usually made with imaging techniques such as echocardiography or CT or MRI. The
first-line treatment is complete surgical resection of the sarcoma and the prognosis is poor in almost all cases.
We present the case of a 67 -year-old male patient that started with a productive cough, hemoptysis, dyspnea
and constitutional syndrome. With postmortem diagnosis of primary undifferentiated heart sarcoma.
\end{abstract}

\section{Puntos destacados}

$\triangleright$ Varón de 67 años de edad con diagnóstico de sarcoma indiferenciado primario de corazón.

$\triangleright$ El diagnóstico patológico y radiológico de esta malignidad suele ser un reto.

$\triangleright$ Tienen un mal pronóstico que se encuentra agravado por la ausencia de un protocolo establecido de tratamiento.

\section{Introducción}

Los sarcomas cardíacos primarios son una entidad patológica rara y constituyen aproximadamente el $1 \%$ de todos los sarcomas de tejidos blandos ${ }^{1}$. Clínicamente, se presentan como falla cardíaca congestiva e invaden el pericardio directamente o por depósito de nódulos en el pericardio visceral ${ }^{2,3}$. En algunos casos, se diagnostican las metástasis en órganos lejanos y sólo se encuentra la lesión primaria incidentalmente ${ }^{4,5}$. El diagnóstico patológico y radiológico de esta malignidad suele ser un reto, ya que estos tumores son muy raros y aún no se reconoce ningún método como estándar de oro diagnóstico ${ }^{6-8}$. No se cuenta con un protocolo establecido de tratamiento, pues estas neoplasias son muy infrecuentes ${ }^{9}$.

Presentamos un caso de un sarcoma indiferenciado primario de corazón con un cuadro clínico caracterizado por tos productiva, hemoptisis, disnea y síndrome constitucional.

\section{Historia clínica y exploración física}

Masculino de 67 años de edad sin antecedentes heredofamiliares de importancia, con antecedente de hipertensión arterial sistémica y etilismo crónico. Con inicio de cuadro clínico de 3 semanas de evolución caracterizado por presencia de tos con expectoración blanquecina y estrías de sangre sin predominio de horario, disnea de medianos a pequeños esfuerzos, ortopnea, astenia, 
adinamia, anorexia y pérdida de peso no intencionada de 20 kg en los últimos 9 meses.

A su ingreso a la unidad de Urgencias presentó signos vitales, tensión arterial (TA) 50/35 mmHg, frecuencia cardíaca (FC) 110 latidos por minuto, frecuencia respiratoria 24 respiraciones por minuto y saturación de oxígeno $\left(\mathrm{SatO}_{2}\right.$ ) del 82\%; por lo que se inició manejo hídrico y oxígeno suplementario a $3 \mathrm{l} / \mathrm{min}$ con posterior mejoría de la TA (110/70 $\mathrm{mmHg}$ ) y SatO 2 (95\%). A la exploración física, el paciente se encontró deshidratado, caquéctico, con palidez generalizada y dificultad respiratoria, neurológicamente con funciones mentales superiores conservadas, isocoria, reflejo fotomotor presente y normal, cuello sin ingurgitación yugular, ausencia de adenopatías, tórax con adecuada amplexión y amplexación, estertores crepitantes subescapulares bilaterales de predominio izquierdo. Ruidos cardíacos rítmicos con adecuada intensidad, con desdoblamiento del segundo ruido, abdomen sin visceromegalias, peristalsis normal, sin dolor a la palpación, extremidades con presencia de edema moderado de ambos miembros pélvicos a nivel subrotuliano.

\section{Pruebas complementarias}

Se solicitaron paraclínicos, dentro de los cuales destacaron: bilirrubina tota $5.15 \mathrm{mg} / \mathrm{dl}$ (0.1-1.0), bilirrubina directa $3.1 \mathrm{mg} / \mathrm{dl}$ (0.2-0.8) y deshidrogenasa láctica 245 U/I (125-240); resto de paraclínicos sin alteraciones.

Dentro de los estudios de imagen, se realizaron ultrasonido de hígado y vías biliares con rastreo a corazón, con hallazgo de imágenes nodulares que modificaban la arquitectura de las cavidades cardíacas, derrame pleural bilateral y presencia de líquido libre intraabdominal, por lo que se solicitó tomografía computarizada de tórax y abdomen, donde se evidenció presencia de múltiples lesiones nodulares a nivel mediastinal y pericárdicas que sugerían, por morfología, lesiones metastásicas de primario desconocido, además de hallazgos observados en la columna vertebral en relación con lesiones de aspecto lítico (Figura 1).

\section{Evolución y diagnóstico}

El paciente presentó datos de choque cardiogénico con presencia de falla ventilatoria que precisó intubación orotraqueal y apoyo vasopresor e inotrópico (norepinefrina + dobutamina). Ante la mala evolución clínica, requirió aumento de parámetros ventilatorios y dosis de norepinefrina, con datos de falla orgánica múltiple, con posterior descenso abrupto de la FC. Los familiares solicitaron libre evolución, y el paciente falleció minutos después.

El reporte de post mórtem por parte del Servicio de Anatomía Patológica fue (Figura 2):

- Corazón con múltiples lesiones nodulares en ambos ventrículos, heterogéneas, café claro que alterna con áreas violáceas y zonas quísticas hemorrágicas.

- Pulmón izquierdo y derecho, así como hígado, con múltiples nódulos metastásicos.

- Neoplasia mesenquimatosa con patrones de crecimiento sólido, alveolar y glandular, con necrosis geográfica extensa, células pequeñas, con núcleo basófilo, vesiculares y escaso citoplasma eosinófilo, que infiltra endocardio así como miocardio.

- Inmunohistoquímica vimentina (+) y (-) para Cro-A, CD99, WT1, AML, MyoD1, PS100, CD56, CD45, CD34, CD31 y D240. Microscopia electrónica, sin hallazgos específicos, FISH del gen EWS sin presentar rearreglo (Figura 3).

- Diagnóstico final de choque cardiogénico secundario a sarcoma indiferenciado primario de corazón.

\section{Discusión y conclusiones}

Los sarcomas cardíacos primarios son una entidad rara que explica alrededor del 20\% de los tumores cardíacos malignos. Usualmente, permanecen asintomáticos hasta que producen efecto de masa, al obstruir el flujo sanguíneo disminuyendo el gasto cardíaco, existe invasión local, embolización o manifestaciones sistémicas. Estos síntomas no son específicos y semejan otras enfermedades cardiovasculares. Los síntomas constitucionales, como fiebre y pérdida de peso, usualmente se asocian con síntomas cardíacos; otros síntomas incluyen tos, hemoptisis, mareo, síncope hasta muerte súbita, como fue el caso de nuestro paciente.

El ecocardiograma bidimensional es actualmente el estudio más usado para detectar tumores cardíacos, sin embargo, ante la inmediata mala evolución clínica de nuestro paciente y el desfavorable desenlace, no se realizó. La tomografía computarizada y la resonancia magnética demuestran con gran precisión la masa tumoral y la extensión a pericardio o vasos
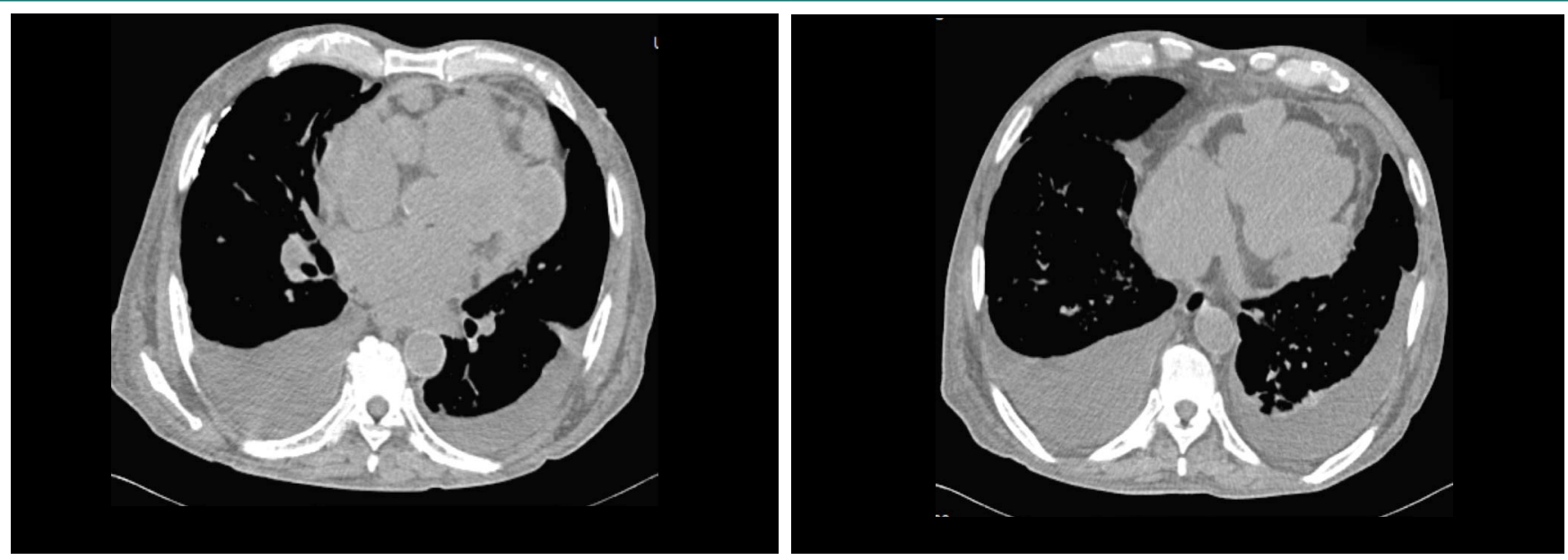

Figura 1. Tomografía computarizada de tórax y abdomen con presencia de múltiples lesiones nodulares a nivel mediastinal y pericardio 

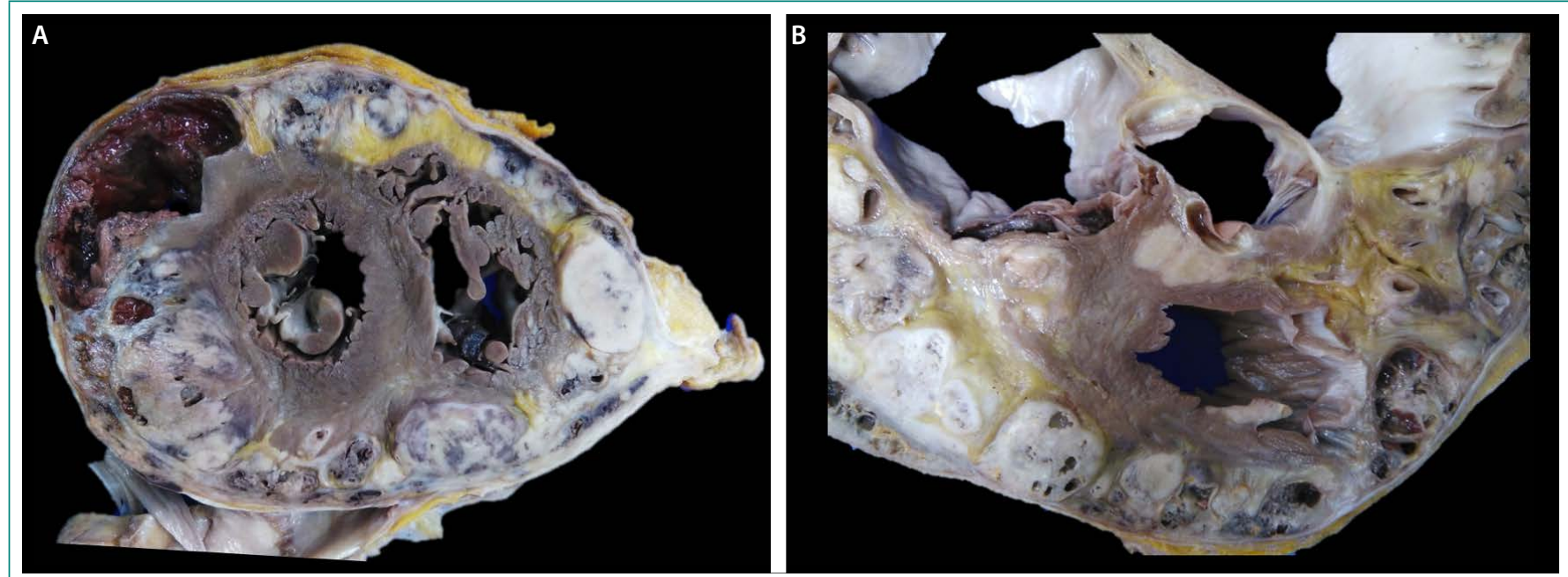

Figura 2. A: corte de corazón, con tumor heterogéneo que se origina del pericardio e infiltra miocardio; con áreas sólidas y quísticas con hemorragia. B: nódulos tumorales infiltrando miocardio

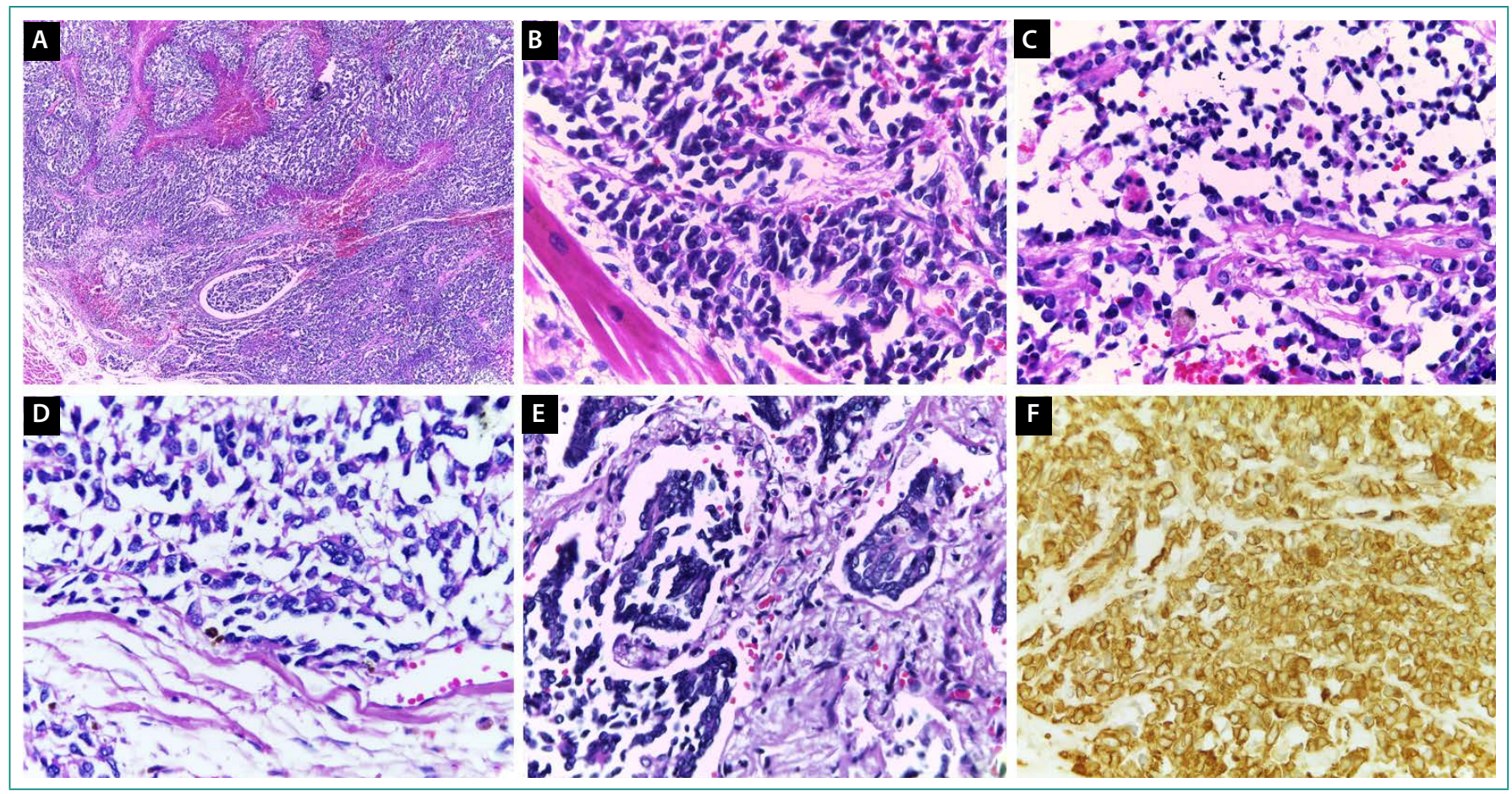

Figura 3. A: neoplasia caracterizada por necrosis geográfica extensa. B: neoplasia maligna mesenquimatosa que diseca a los miocardiocitos. C: células pequeñas, redondas y basófilas. D: células con citoplasma y escaso eosinófilo, con núcleo grande, pleomórfico, con cromatina grumosa y nucleolo evidente. E: células neoplásicas dentro de alvéolos y disecando el parénquima pulmonar. F: anticuerpo vimentina con positividad intensa y difusa en células neoplásicas

de gran calibre. En términos de diagnóstico diferencial, a veces puede ser difícil distinguir el sarcoma bien diferenciado del lipoma, basándose únicamente en las técnicas de imagen actuales, ya que, además, ambos tienen un patrón de apariencia nodular. Sin embargo, el sarcoma, a diferencia del lipoma, generalmente no está encapsulado y tiene un comportamiento infiltrativo. Otros hallazgos más orientados hacia el sarcoma comprenden apariencia no homogénea a pesar de la presencia de una porción grasa intralesional, dimensiones más grandes y componente de tejido blando más prominente.

La resección quirúrgica completa continúa siendo el tratamiento primario de elección. En caso de difícil accesibilidad anatómica, la resección con autotras- plante ofrece mejores resultados. No obstante, los resultados son paliativos, ya que se observa recurrencia y metástasis en la mayoría de los casos. A pesar del tratamiento, el pronóstico continúa siendo desfavorable.

\section{Bibliografía}

Mercado-Guzmán MP, Meléndez-Ramírez G, Castillo-Castellón F, KimuraHayama E. Evaluación de tumores cardíacos por tomografía computada multidetector y resonancia magnética cardíaca. Arch Cardiol Mex. 2016 Oct-Dec; 86(4): 335-349. doi: 10.1016/j.acmx.2016.04.005. 
2. Nael A, Johnston A, Dacosta-lyer M, Deshmukh-Reane S, Ouyang Y, Nael K, et al. An intimal cardiac sarcoma with overexpression of MDM2, first presented as intestinal obstruction, a case report and review of literature. Am Soc Clin Pathol Case Rep. 2014; 14(4): 2-19.

3. Ramlawi B, Leja MJ, Abu Saleh WK, Al Jabbari O, Benjamin R, Ravi V, et al. Surgical treatment of primary cardiac sarcomas: review of a single-institution experience. Ann Thorac Surg. 2016 Feb; 101(2): 698-702. doi: 10.1016/j. athoracsur.2015.07.087.

4. Diab MS, Smelt J, Fletcher N, Sarsam M. Cardiac sarcoma presenting with abdominal pain and mimicking myxoma on echocardiogram. JRSM Open. 2016 Sep 1; 7(9): 2054270416649286. doi: 10.1177/2054270416649286.

5. Byon JH, Kwak HS, Chung GH, Jang KY. Acute stroke from tumor embolus in a patient with cardiac sarcoma: aspiration thrombectomy with penumbra catheter. Interv Neuroradiol. 2016 Feb; 22(1): 88-90. doi $10.1177 / 1591019915609782$
6. Chawla SP, Papai Z, Mukhametshina G, Sankhala K, Vasylyev L, Fedenko A, et al. First-line aldoxorubicin vs doxorubicin in metastatic or locally advanced unresectable soft-tissue sarcoma: a phase $2 \mathrm{~b}$ randomized clinical trial. JAMA Oncol. 2015 Dec; 1(9): 1272-1280. doi: 10.1001/jamaoncol.2015.3101.

7. Lima PP, López-Almodóvar LF, Jiménez Jl, Orrade JL, Cañas A. Sarcoma cardíaco primario que comienza como dolor abdominal e isquemia intestinal: a propósito de un caso y revisión de la literatura. Cirugía Cardiovascular. 2013; 20(3): 156-158. doi: 10.1016/j.circv.2013.05.006

8. Cannavale G, Francone M, Galea N, Vullo F, Molisso A, Carbone I. Fatty images of the heart: spectrum of normal and pathological findings by computed tomography and cardiac magnetic resonance imaging. Biomed Res Int. 2018 Jan 9; 2018: 5610347. doi: 10.1155/2018/5610347.

9. Li H, Yang S, Chen H, Yang Z, Hong T, Hou Y, et al. Survival after heart transplantation for non-metastatic primary cardiac sarcoma. J Cardiothorac Surg. 2016; 11: 145. doi: 10.1186/s13019-016-0540-x. 